time existing in East Anglia, and broken up by the irruption of the sea into the area in Crag times. At Bawdsey, on the Suffolk coast, the sea is to-day attacking a cliff composed of London clay, Red Crag, Glacial Gravel and surface material. As these various beds are washed away, the heavier contents collect at low tide mark, and are forming a deposit similar in some respects to the Suffolk Bone Bed. It is indeed highly probable that this was accumulated under analogous conditions, and it is possible that the mammalian remains of different ages in the Bone Bed, together with the flint implements, at one time occupied their respective geological horizons in the now vanished land of Suffolk.

The mammalian bones and teeth in the Suffolk Bone Bed range from Upper Miocene to late Pliocene times, when this deposit was laid down. It is possible that the earliest group of the sub-crag flint implements may date back to the earlier part of the
Pliocene, or even to more ancient times, but this is not yet established. The specimens of the earliest group, though exhibiting many archaic characteristics, cannot however be looked upon as the type of artefact likely to be made by the earliest representatives of the human race; but this group is obviously of an extreme antiquity, and its existence points to a much greater age for the human race than has hitherto been supposed. It has long been recognised and accepted that man was present in East Anglia before the deposition of the Red Crag some 500,000 years ago, but it now appears that this period of time must be greatly extended to include the earliest group of the pre-Crag artefacts.

The excavations which over a number of years have been conducted by Mr. Reid Moir in the Suffolk Bone Bed, have been made possible by the invaluable financial help of the Royal Society, the Percy Sladen Memorial Fund and Mr. T. R. Parkington of Ipswich.

\title{
Artificial Production of the Hormone of the Corpus Luteum
}

$\mathrm{T}$

$\mathrm{HE}$ transformations of cholesterol into the male sex hormone (androsterone) and of stigmasterol into a crude product having the biological activity of the corpus luteum hormone ${ }^{1}$ have been rapidly followed by further important developments in this field of investigation of the sex hormones, and the corpus luteum hormone has now been prepared in a chemically pure state from stigmasterol and also from pregnandiol.

The conversion of pregnandiol into the hormone was achieved by Butenandt and Schmidt ${ }^{2}$ in a strikingly simple manner. Having first shown that by partial hydrolysis of pregnandiol diacetate the nuclear acetoxy group was hydrolysed, they converted the monoacetate by a series of stages into the hormone, and showed thereby that the hormone was the $\Delta^{1: 2}$ or $\Delta^{\mathbf{4} 5}$ unsaturated diketone corresponding with pregnandiol. These authors then found that pregnandiol could be transformed into the pure hormone by three simple stages, namely, oxidation to pregnandione, monobromination, and subsequent elimination of hydrogen bromide by heating with pyridine. As pregnandiol may be isolated from the urine of pregnancy without difficulty, there is no doubt that considerable supplies of the artificial hormone will be manufactured by this method.

The isolation of the pure hormone from the degradation products of stigmasterol has been recorded by Fernholz ${ }^{3}$ and by Butenandt and Westphal ${ }^{4}$. As Fernholz and Chakravorty ${ }^{5}$ have also shown that both cholesterol and stigmasterol may be degraded to the same 3-hydroxy-nor-allocholanic acid, this conversion of stigmasterol into the corpus luteum hormone establishes the positions 3 and 20 for the two keto groups of the hormone. The $\triangle^{4: 5}$ position of the double bond is also regarded by Butenandt and Westphal as established by the formation of the hormone from stigmasterol, but since the position of the double bond of stigmasterol is based only on analogy with cholesterol ${ }^{6}$ it is better to admit that the $\Delta^{1: 2}$ position for the double bond of the hormone has not been rigorously excluded.

Butenandt and $\mathrm{Mamoli}^{7}$ have directed attention to the fact that pregnandiol is therefore a hydrogenation product of the hormone, and in the light of this conception it is easy to understand the presence of large quantities of pregnandiol in the urine during pregnancy, for the diol is thus seen to be the form in which the hormone is excreted, just as cholesterol is exereted in the form of its hydrogenation product, coprosterol.

The artificial hormone, like that prepared from corpus luteum extracts, exists in two polymorphous forms, one of which is readily converted into the other8.

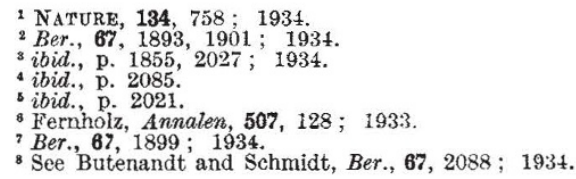

\section{Temperatures of the Stars}

IN a lecture before the Newcastle-on-Tyne Astro1 nomical Society on December 12, Mr. W. M. H. Greaves described the way in which stellar temperatures are derived from a study of the spectra of stars.

All information regarding temperatures of the stars is derived from their light and its analysis. In heating a metal, while at first the radiation is almost entirely limited to the infra-red, with increase of temperature it includes wave-lengths in the visible part of the spectrum, and the proportion of blue to red light emitted increases as the temperature rises. But we cannot generally find temperature from colour, since the emissivity of bodies varies. Nevertheless, our knowledge of the temperature of stars is derived from measures of the colour of star light, the source of which is the outer layers of the star.

The 'black body', which theoretically absorbs all radiation falling on it and which, when heated, emits radiation of all kinds, is taken as a standard of reference. For such a body the proportions of emitted light at different wave-lengths are connected with temperature by Planck's formulæ. Observation shows that, so far as measurement made between spectral lines is concerned, stars are emitting radiation in a 